\title{
Approach to undifferentiated dyspnea in emergency department: aids in rapid clinical decision-making
}

\author{
Siva Nageswara Rao Guttikonda* and Kiran Vadapalli
}

\begin{abstract}
Background: Diagnosis and management of patients presenting with acute dyspnea is one of the major challenges for physicians in emergency department (ED). A correct diagnosis is frequently delayed and difficult to ascertain, and clinical uncertainty is common, explaining the need for rapid diagnosis and a management plan. The primary aim of our study is to assess a diagnostic strategy using multiorgan point of care ultrasonography (USG) to differentiate patients presenting with acute dyspnea to ED into different diagnostic categories for timely management in a resource-limited setting.

Methods: This is a prospective cohort study which assessed the diagnostic performance of a strategy in evaluating patients presenting with undifferentiated dyspnea as primary predominant complaint to ED. Focused multiorgan USG which includes cardiac USG for left ventricle systolic function, right ventricle enlargement, and pericardial effusion, inferior vena cava (IVC) diameter and collapsibility, lung USG to identify various patterns (acute interstitial syndrome, pneumothorax, pleural effusion, consolidation, etc.) and renal USG to assess kidney size and echotexture was performed. Later, patients were grouped into one of ten clinical syndromes defined in the study based on USG and clinical patterns. Emergency diagnosis was compared with final hospital diagnosis to assess the accuracy of this strategy.

Results: Concordance between ED diagnosis of dyspnea using the diagnostic strategy proposed in the study with final hospital diagnosis was high with agreement in $88 \%$ of patients (Kappa statistic $=.805, p=.000$ ) which is statistically significant. The most common diagnosis was acute decompensated heart failure (ADHF). Sensitivity and specificity of the diagnostic strategy used in this study to identify ADHF was 97.3 and 93.3\%, respectively. On multivariate analysis, jugular venous distension, fever and cough, ejection fraction (by eyeball method), dilated IVC, absent to decreased lung sliding showed independent association in predicting cardiac and non-cardiac diagnosis.
\end{abstract}

Conclusions: The present study concludes that integrating focused multiorgan USG by lung-cardiac-IVC and renal ultrasound into routine clinical evaluation of patients with dyspnea has a higher accuracy for differentiating causes of dyspnea in emergency department. This strategy can be adopted even in resource limited setting.

Keywords: Dyspnea, Multiorgan USG, Emergency department, Diagnostic strategy, Resource limited setting

\section{Background}

Acute dyspnea is one of the main reasons for admission to the emergency department (ED) [1]. Physicians working in the ED often need to make a rapid diagnosis and devise a treatment plan on the basis of limited clinical information $[2,3]$. Rapid and accurate diagnosis and management can be lifesaving for patients with acute dyspnea [4]. However,

\footnotetext{
* Correspondence: sivanagdr@gmail.com

Department of Internal Medicine, Rangaraya Medical College, Government General Hospital, Raja Ram Mohan Rai road, Kakinada, Andhra Pradesh 533001, India
}

making a differential diagnosis and selecting early treatment for patients with acute dyspnea in the ED is a clinical challenge that requires complex decision-making in order to achieve hemodynamic balance, improve functional capacity, and decrease mortality and the length of hospital stay [5]. Methods for evaluation of emergency patients with possible acute decompensated heart failure (ADHF) include the history, physical examination, chest radiography, 12-lead electrocardiography (ECG), and measurement of brain natriuretic peptide (BNP) or $\mathrm{N}$-terminal pro-BNP [6]. The physical examination, even with the 
addition of chest radiography and ECG, is often imprecise and simply starting "dual therapy" for ADHF and chronic obstructive pulmonary disease (COPD) can be harmful [7].

Recently, there has been interest in utilizing chest USG for the diagnosis of acute respiratory failure in ICU. Bedside Lung USG in Emergency (BLUE) protocol proposed by Lichenstein et al. is a landmark study in this regard [8]. There have been few ED studies demonstrating the role of multiorgan point of care USG to evaluate dyspnea. Kajimoto et al. were the first to demonstrate the screening potential of rapid evaluation by lung-cardiac-inferior vena cava (LCI) integrated ultrasound for differentiating ADHF from primary pulmonary disease in patients with acute dyspnea in the emergency setting [9]. Russel et al. used LUCUS protocol to diagnose ADHF in patients with undifferentiated dyspnea in ED [10]. Later, Pirozzi et al. and Gallard et al. evaluated adding cardiopulmonary USG to routine clinical examination to manage dyspnea patients in ED [11, 12]. Most of the above studies focused on assessing the diagnostic performance of USG in identifying ADHF in patients presenting with dyspnea to ED, leaving behind non-cardiac causes of dyspnea, thereby necessitating a strategy to differentiate various non-cardiac causes of dyspnea. No studies were performed in resource-limited ED setting. The primary aim of our study was to assess a diagnostic strategy using multiorgan point of care ultrasonography (USG) to distinguish patients presenting with acute dyspnea to ED into different diagnostic categories for timely management in a resource-limited setting.

\section{Methods}

\section{Study design and setting}

This was a prospective cohort study which assessed the diagnostic performance of a strategy using multiorgan point of care USG in evaluating patients presenting with dyspnea in emergency care. This study was done at emergency department (ED) at Government General Hospital, Kakinada, between June 2016 and December 2016. This study protocol was approved by the Institutional Ethical Committee. A written informed consent was obtained from all patients.

\section{Selection of patients}

Patients who were 16 years and older, visiting the ED with undifferentiated dyspnea as primary predominant complaint (either sudden onset dyspnea or increase in severity of chronic dyspnea) were included in the study within $1 \mathrm{~h}$ of their arrival. Undifferentiated dyspnea was defined for the purpose of this study as two or more possible etiologies in the differential diagnosis according to their treating clinician. Patients with definite etiology of dyspnea which would include a patient with known heart failure not compliant with medication or diet restrictions, or known asthmatics who responded to bronchodilators as per the treating clinician, or patients in whom the treating clinicians were confident in their diagnoses after initial assessment (e.g., patients in whom ECG shows ST elevation MI) were excluded.

\section{Clinical evaluation}

Patient's medical history, vital signs, and systemic examination were recorded by the enrolling physician. Dyspnea at admission was measured using a 5-point LIKERT scale in sitting position. All patients underwent routine tests as part of the hospital protocol (ECG, chest X-ray, and labs). The clinical probability of heart failure and obstructive lung disease was noted using modified Boston criteria and GOLD clinical criteria of COPD, respectively [13-15].

\section{Multiorgan point-of-care USG}

After initial evaluation, all patients were subjected to a focused multiorgan USG at bedside using a standard medium frequency curved array probe which includes the following.

\section{Cardiac USG}

Parasternal long- and short-axis views-left ventricular ejection fraction (EF) was estimated visually in the parasternal long-axis view by wall contraction and thickening. EF was confirmed in the parasternal short-axis view at the level of the papillary muscles. Mitral valve E-point septal separation (EPSS) is the distance from the anterior mitral valve leaflet, and the ventricular septum in early diastole measured in $M$ mode is also noted for every patient. Left ventricular systolic function was typically graded as normal (EF > 50\%), moderate dysfunction (EF 30-50\%), or severe dysfunction $(\mathrm{EF}<30 \%)$ basing on eyeball visual estimate and EPSS measurement. A qualitative evaluation of the right ventricle (RV) dimension was made for RA/RV dilatation, considering RV/LV-end diastolic diameter $>0.9$ in the AP4 view as abnormal.

\section{Lung USG}

Lung USG was performed on each hemithorax divided into five zones (two anterior, two lateral, and one posterior) with patient in seated or lying down position. Several signs were explored to conclude on typical patterns as per the international evidence-based recommendations on pointof-care lung USG [16] which includes lung sliding, pleural effusion (anechoic space between parietal and visceral pleura with sinusoid sign), pneumothorax (loss of lung sliding with positive lung point), and acute interstitial syndrome (AIS), defined as B-pattern with at least three B-lines in two lung zones bilaterally and lung consolidation 
signs (focal B-lines with tissue-like echotexture and dynamic air bronchograms) [16, 17].

\section{IVC USG}

IVC USG was performed in sub-xiphoid view for diameter and collapsibility. An IVC with a maximal diameter of $\geq$ $2 \mathrm{~cm}$ and $<50 \%$ collapse was considered plethoric. An IVC with a maximal diameter of $\leq 2 \mathrm{~cm}$ and $>50 \%$ collapse was considered collapsible.

\section{Renal USG}

Renal USG was performed using an anterior subcostal approach using the liver as a sonographic window for the right kidney and an intercostal approach for the left kidney. Maximal longitudinal axis length and echogenicity of the kidneys were noted.

After a complete assessment, patients were assigned into one of the ten clinical syndromes defined as mentioned in Table 1 by following the constructed algorithm mentioned in Fig. 1. Patient's time for improvement in their symptom status was noted.

The final hospital diagnosis of patients was determined by two independent physicians, a cardiologist and a pulmonologist, using the reference standard definition for heart failure and pulmonary diseases in accordance with routine standard evaluation using chest X-ray, echocardiographic examination, cardiac functional assessment (exercise test), pulmonary function test, full blood count, biochemistry, and invasive investigation or angiography without knowledge of the USG data collected in ED. The initial ED diagnosis is compared with final hospital diagnosis as mentioned on discharge sheet.

\section{Statistical analysis}

All data were analyzed using the SPSS V.20.0.0 software package. A $P$ value $<0.05$ is regarded as statistically significant. The comparison of the group of patients who have cardiac and non-cardiac diagnosis was done using Student's $t$ test and chi-square test for continuous and categorical variables, respectively. The performance of different diagnostic tools was analyzed using sensitivity and specificity analysis. The level of agreement between ED diagnosis and final hospital diagnosis was assessed using Cohen's Kappa test.

\section{Results and discussion}

We enrolled a total of 108 patients in the study. Of these, 8 patients were excluded as they left the hospital against advice. So, a total of 100 patients were included in the data analysis.

\section{Patient characteristics}

The mean $( \pm$ SD) age among dyspnea patients was $50( \pm$ $15.85)$ years with a minimum age of 16 years and maximum age of 90 years. Age stratified ratio according to $\geq$ $60:<60$ years is $36: 72$ showing elderly patients constituted only $36 \%$ of our sample. All the patients in our study were categorized into three groups basing on the diagnosis. The groups were cardiac, non-cardiac (includes pulmonary, renal, and metabolic/systemic causes), and with combined cardiac and non-cardiac cause of dyspnea. Distribution of our patients according to category of diagnosis was cardiac constituting $43 \%$, non-cardiac $29 \%$, and patients with both cardiac and non-cardiac conditions $28 \%$. The mean age of presentation of cardiac patients was

Table 1 Clinical syndromes basing on USG patterns and clinical variables

\begin{tabular}{|c|c|}
\hline $\begin{array}{l}\text { ADHF } \\
\text { (systolic dysfunction) }\end{array}$ & Acute interstitial syndrome (the presence of multiple diffuse bilateral B-lines) with LV dysfunction on cardiac USG \\
\hline Acute pneumonia & $\begin{array}{l}\text { Lung USG showing focal B-lines, subpleural echo poor region with tissue-like echotexture and dynamic air } \\
\text { bronchogram with normal LV function. }\end{array}$ \\
\hline ARDS & $\begin{array}{l}\text { Acute interstitial syndrome (presence of multiple diffuse bilateral B-lines) in non-homogenous distribution and } \\
\text { anterior subpleural consolidation, reduced lung sliding normal LV function with suggestive clinical presentation } \\
\text { (sudden onset and suggestive sepsis). }\end{array}$ \\
\hline $\begin{array}{l}\text { COPD or Obstructive } \\
\text { airway disease }\end{array}$ & $\begin{array}{l}\text { Bilateral A-lines with decreased lung sliding on lung USG with normal LV function and normal kidneys with key } \\
\text { indicators of COPD present clinically. }\end{array}$ \\
\hline $\begin{array}{l}\text { Acute Pulmonary } \\
\text { Embolism }\end{array}$ & $\begin{array}{l}\text { Normal LV function, A-lines on lung USG with or without focal B-lines, dilated RV, dilated IVC with low collapsibility } \\
\text { index with high pre-test probability of PE. }\end{array}$ \\
\hline $\begin{array}{l}\text { Chronic lung disease } \\
\text { (ILD or chronic lung fibrosis) }\end{array}$ & $\begin{array}{l}\text { Normal LV function, irregular fragmented pleural line, subpleural abnormalities, bilateral B-lines in non- homogenous } \\
\text { distribution with or without dilated RV and dilated IVC with low collapsibility index with suggestive clinical picture } \\
\text { (h/o chronic exertional dyspnea and cough). }\end{array}$ \\
\hline Diastolic Heart Failure & $\begin{array}{l}\text { Normal LV function with LV hypertrophy (LVH), left atrial enlargement, multiple diffuse bilateral B-lines, dilated IVC } \\
\text { with low collapsibility index with suggestive clinical presentation (e.g., hypertensive, diabetic) }\end{array}$ \\
\hline Volume overload & Normal LV function, normal RV, multiple diffuse bilateral B-lines, dilated IVC, contracted kidneys. \\
\hline Pneumothorax & $\begin{array}{l}\text { Normal LV function, bilateral A-lines with absent lung sliding with observed lung point with } \\
\text { suggestive clinical picture. }\end{array}$ \\
\hline Tamponade & $\begin{array}{l}\text { Normal LV function, pericardial effusion with dilated and non-collapsible IVC and RA/RV } \\
\text { diastolic collapse with suggestive clinical signs. }\end{array}$ \\
\hline
\end{tabular}




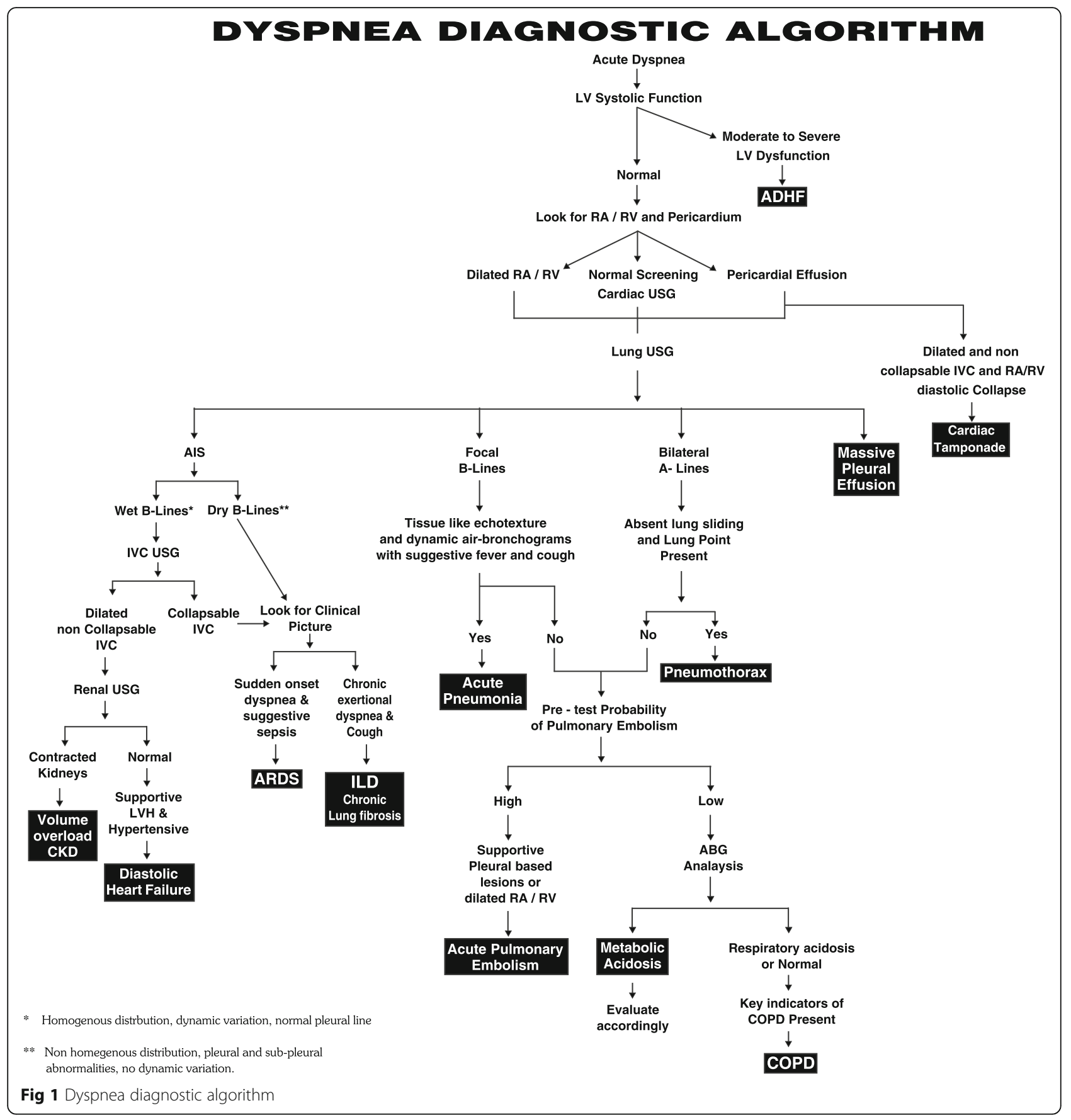

$53( \pm 16.16)$ years, and non-cardiac patients was $45( \pm 14.05)$ years (Table 2).

\section{Distribution of patients according to diagnosis}

Patients with ADHF constituted 43\%, and patients having both cardiac and non-cardiac cause of dyspnea constituted $28 \%$ mostly with a diagnosis of ischemic cardiomyopathy (ICMP) with chronic kidney disease (CKD), COPD, or interstitial lung disease (ILD) with RV failure, and ADHF with pneumonia. The remaining patients had other causes like ARDS (7\%), COPD (4\%), acute pulmonary embolism (7\%), acute pneumonia (4\%), volume overload (4\%), and massive pleural effusion (3\%) (Table 3).

On univariate analysis of clinical variables recorded at the time of admission, history of orthopnea, fever and cough, past history of CAD, jugular venous distension, and displaced apex beat, modified Boston criteria for HF score showed significant difference between the diagnostic categories. Among the patients with absent to decreased lung sliding, $77 \%$ belonged to non-cardiac group and $23 \%$ 
Table 2 Characteristics of patients according to category of diagnosis (continuous variables) ( $N=$ cardiac 43 , non-cardiac 29, both 28)

\begin{tabular}{|c|c|c|c|c|}
\hline & $\begin{array}{l}\text { Cardiac patients mean } \pm \text { SD and } \\
(95 \% \mathrm{Cl})\end{array}$ & $\begin{array}{l}\text { Non-cardiac patients mean } \pm \text { SD and } \\
(95 \% \mathrm{Cl})\end{array}$ & $\begin{array}{l}\text { Both mean } \pm \text { SD and } \\
(95 \% \mathrm{Cl})\end{array}$ & $P$ value \\
\hline Age & $\begin{array}{l}53.55 \pm 16.16 \\
(48.58-58.05)\end{array}$ & $\begin{array}{l}45.31 \pm 14.05 \\
(39.36-50.6)\end{array}$ & $\begin{array}{l}49.53 \pm 16.31 \\
(43.2-55.8)\end{array}$ & 0.093 \\
\hline Systolic blood pressure & $\begin{array}{l}139.302 \pm 31.04 \\
(129.74-148.85)\end{array}$ & $\begin{array}{l}119.65 \pm 25.28 \\
(110.03-129.27)\end{array}$ & $\begin{array}{l}143.57 \pm 45.88 \\
(125.78-161.63)\end{array}$ & 0.002 \\
\hline Diastolic blood pressure & $\begin{array}{l}85.81 \pm 15.77 \\
(80.96-90.68)\end{array}$ & $\begin{array}{l}74.82 \pm 13.26 \\
(69.78-79.87)\end{array}$ & $\begin{array}{l}91.42 \pm 27.31 \\
(80.83-102.02)\end{array}$ & 0.005 \\
\hline Heart rate & $\begin{array}{l}111.465 \pm 20.83 \\
(105.03-117.08)\end{array}$ & $\begin{array}{l}117.00 \pm 31.15 \\
(105.14-128.24)\end{array}$ & $\begin{array}{l}113.89 \pm 21.53 \\
(105.54-122.24\end{array}$ & 0.642 \\
\hline Respiratory rate & $\begin{array}{l}28.44 \pm 4.22 \\
(27.14-29.74)\end{array}$ & $\begin{array}{l}33.138 \pm 6.610 \\
(30.62-35.65)\end{array}$ & $\begin{array}{l}30.536 \pm 3.2028 \\
(29.29-31.77)\end{array}$ & 0.00 \\
\hline $\mathrm{SpO} 2$ & $\begin{array}{l}89.93 \pm 8.90 \\
(87.18-92.62)\end{array}$ & $\begin{array}{l}82.964 \pm 13.16 \\
(77.85-88.07)\end{array}$ & $\begin{array}{l}80.82 \pm 14.46 \\
(75.21-86.42)\end{array}$ & 0.004 \\
\hline IVC maximum diameter & $\begin{array}{l}18.91 \pm 3.87 \\
(17.71-20.10)\end{array}$ & $\begin{array}{l}10.46 \pm 6.89 \\
(7.84-13.08)\end{array}$ & $\begin{array}{l}17.17 \pm 5.147 \\
(15.17-19.16)\end{array}$ & 0.00 \\
\hline IVC minimum diameter & $\begin{array}{l}12.86 \pm 4.53 \\
(11.46-14.25)\end{array}$ & $\begin{array}{l}5.26 \pm 5.50 \\
(3.17-7.36)\end{array}$ & $\begin{array}{l}11.06 \pm 5.993 \\
(8.74-13.38)\end{array}$ & 0.00 \\
\hline LV systolic diameter in PLAX & $\begin{array}{l}3.85 \pm 1.213 \\
(3.48-4.22)\end{array}$ & $\begin{array}{l}2.82 \pm 0.583 \\
(2.60-3.04)\end{array}$ & $\begin{array}{l}3.56 \pm 0.975 \\
(3.18-3.94)\end{array}$ & 0.00 \\
\hline EPSS & $\begin{array}{l}12.007 \pm 6.80 \\
(9.912-14.10)\end{array}$ & $\begin{array}{l}3.624 \pm 1.70 \\
(2.97-4.27)\end{array}$ & $\begin{array}{l}9.039 \pm 5.93 \\
(6.73-11.34)\end{array}$ & 0.00 \\
\hline Kidney size & $\begin{array}{l}9.60 \pm 0.667 \\
(9.39-9.80)\end{array}$ & $\begin{array}{l}9.57 \pm 1.36 \\
(9.05-10.05)\end{array}$ & $\begin{array}{l}8.96 \pm 1.16 \\
(8.50-9.41)\end{array}$ & 0.031 \\
\hline Blood urea nitrogen & $\begin{array}{l}39.69 \pm 13.06 \\
(35.67-43.71)\end{array}$ & $\begin{array}{l}57.65 \pm 43.71 \\
(41.02-74.28)\end{array}$ & $\begin{array}{l}65.42 \pm 30.97 \\
(53.41-77.43)\end{array}$ & 0.001 \\
\hline Serum creatinine & $\begin{array}{l}1.295 \pm 0.66 \\
(1.09-1.51)\end{array}$ & $\begin{array}{l}3.093 \pm 3.58 \\
(1.72-4.45)\end{array}$ & $\begin{array}{l}3.476 \pm 2.669 \\
(2.46-4.53)\end{array}$ & 0.00 \\
\hline Time to relief from dyspnea in $\mathrm{Hrs}$ & $\begin{array}{l}22.64 \pm 18.85 \\
(16.36-28.93)\end{array}$ & $\begin{array}{l}18.00 \pm 21.015 \\
(4.64-31.35)\end{array}$ & $\begin{array}{l}32.00 \pm 21.91 \\
21.09-42.90)\end{array}$ & 0.139 \\
\hline Likert scale & $\begin{array}{l}2.816 \pm 0.729 \\
(2.57-3.05)\end{array}$ & $\begin{array}{l}2.87 \pm 1.027 \\
(2.26-3.45)\end{array}$ & $\begin{array}{l}3.3 \pm 0.656 \\
(2.99-3.60)\end{array}$ & 0.075 \\
\hline Hospital LOS & $\begin{array}{l}6.034 \pm 3.109 \\
(5.07-6.99)\end{array}$ & $\begin{array}{l}4.86 \pm 4.434 \\
(3.22-6.51)\end{array}$ & $\begin{array}{l}7.56 \pm 5.998 \\
(5.24-9.89)\end{array}$ & 0.01 \\
\hline Modified Boston Criteria for HF & $\begin{array}{l}8.07 \pm 1.334 \\
(7.65-8.48)\end{array}$ & $\begin{array}{l}6.86 \pm 1.156 \\
(6.442-7.302)\end{array}$ & $\begin{array}{l}7.893 \pm 1.065 \\
(7.48-8.306)\end{array}$ & 0.00 \\
\hline
\end{tabular}

SD standard deviation, $C l$ confidence interval, IVC inferior vena cava, $L V$ left ventricle, EPSS E-point septal separation, LOS length of stay, $H F$ heart failure

belong to cardiac group which is significant difference between the diagnostic groups $(p=.002)$. B-profile did not show significant difference between the groups. It was observed in $72 \%$ of cardiac patients while $41 \%$ of non-cardiac patients also showed B-profile. EF visual estimate correlated well with final diagnosis with only 2 non-cardiac patients given false positive LV dysfunction (Table 4). IVC diameter between cardiac (mean $18.91 \pm$ $3.87 \mathrm{~mm}$ ) and non-cardiac groups (mean $10.46 \pm 6.89 \mathrm{~mm}$ ) showed significant difference (Table 2). On multivariate analysis, only jugular venous distension, ejection fraction (by eyeball method), dilated IVC (for cardiac), h/o fever and cough, and absent to decreased lung sliding (for non-cardiac) showed independent association in predicting cardiac and non-cardiac diagnosis.

\section{Concordance between emergency diagnosis and final diagnosis}

The concordance between initial at admission diagnosis and final hospital diagnosis at discharge was analyzed by Cohen's Kappa test. There is agreement in diagnosis in $88 \%$ of patients. The measure of agreement Kappa $=0.805$ $(p=.000)$. The level of agreement between emergency diagnosis using our diagnostic strategy and final hospital diagnosis is statistically significant.

\section{Discussion}

Our study shows 60 out of 68 patients (88\%) received correct disease-specific treatment using the above diagnostic strategy with agreement in ED diagnosis and final discharge diagnosis. The measure of agreement Kappa is 
Table 3 Distribution of patients according to diagnostic category

\begin{tabular}{|c|c|c|c|}
\hline Emergency department diagnosis & Number of patients & Number of incorrect ED diagnosis & Final discharge diagnosis of incorrect ED diagnosis cases \\
\hline $\begin{array}{l}\text { ADHF } \\
\text { (systolic or diastolic heart failure) }\end{array}$ & 43 & 2 & $\begin{array}{l}\text { 1. Volume overload/AKI } \\
\text { 2. Acute bilateral bronchopneumonia }\end{array}$ \\
\hline COPD & 4 & Nil & \\
\hline ARDS & 7 & 1 & Anaphylaxis \\
\hline Acute pulmonary embolism & 7 & 4 & $\begin{array}{l}\text { 1. ASD/Beri beri } \\
\text { 2. Pneumonia/sepsis with DIC } \\
\text { 3. ADHF/pneumonia/severe anemia } \\
\text { 4. ILD with Ac exacerbation of PAH }\end{array}$ \\
\hline Acute pneumonia & 4 & 1 & Severe anemia with ADHF \\
\hline Volume overload (AKI, CKD) & 4 & Nil & \\
\hline Massive pleural effusion & 3 & Nil & \\
\hline Both (cardiac and non-cardiac) & 28 & 3 & $\begin{array}{l}\text { 1. IPF acute exacerbation/RV failure } \\
\text { 2. ADHF/malignant effusion } \\
\text { 3. Severe anemia/RPGN }\end{array}$ \\
\hline
\end{tabular}

ADHF acute decompensated heart failure, $A K I$ acute kidney injury, $A S D$ atrial septal defect, COPD chronic obstructive pulmonary disease, DIC disseminated intravascular coagulation, $A R D S$ acute respiratory distress syndrome, ILD interstitial lung disease, $P A H$ pulmonary arterial hypertension, IPF idiopathic pulmonary fibrosis, RPGN rapidly progressive glomerulonephritis

0.805 ( $p$.000) where the level of agreement is statistically significant. Patients who expired were excluded for evaluation of diagnostic strategy as many of these expired within $48 \mathrm{~h}$ of presentation and were not able to complete their in-hospital evaluation. Most of the discrepancies occurred in patients with acute RV failure if it was due to an acute event like pulmonary embolism or acute exacerbation of chronic pulmonary artery hypertension and in patients having additional component of COPD in ADHF patients. Two studies published in 2014, similar to the present study, assessed the impact of multi-organ POCUS, in addition to history and physical examination, on the accuracy of treating the patient. In a randomized controlled trial (RCT) where patients were randomly assigned to initial assessment with and without point-of-care ultrasonography (POCUS), Pirozzi et al. found that the rate of discordance between initial and final diagnosis was $5 \%$ in the POCUS group compared to $50 \%$ in the control group [11]. Lauresen et al. found a proportion of correct presumptive diagnosis in the POCUS group of $88 \%$ compared to $63.7 \%$ in the control group, a significant difference [18].

The most common diagnosis for dyspnea in this study was ADHF (43\%). Sensitivity and specificity of the diagnostic strategy used in this study to identify ADHF was 97.3 and 93.3\%, respectively. With regard to the test performance characteristics of POCUS as a stand-alone test for ADHF, Kajimoto et al. found a sensitivity and specificity of 94 and 91\% [9] and Russell et al. reported sensitivity and specificity of 83 and $83 \%$ [9, 10]. As opposed to the more comprehensive and time-consuming echocardiography protocols used by other investigators $[19,20]$, the echocardiography component of our study protocol simply focused on ejection fraction by gross visual estimation [21, 22] (an adopted method by ACEP for emergency cardiac USG to assess global LV systolic function), presence or absence of pericardial effusion, and right ventricular enlargement while in previous studies, they evaluated diastolic function and Doppler evaluation of the heart. To diagnose diastolic heart failure, we have taken a set of parameters like LVH, LA enlargement, bilateral B-lines on lung USG, and dilated IVC along with suggestive clinical signs. Similarly, the lung examination consisted of assessment of ten zones bilaterally. We have attained similar sensitivity and specificity using this abbreviated protocol. Not only is such an abbreviated protocol feasible during initial resuscitation of the sickest dyspneic patients, but it is likely to be more generalizable to non-expert sonographers across all settings.

To date, there have been just a few studies evaluating a multi-organ POCUS protocol similar to our studycombining abbreviated echocardiography, lung USG, and IVC assessment in the setting of undifferentiated dyspnea. In addition, we included renal USG in the present study to assess kidney size and echotexture. The majority of these studies focused strictly on diagnosis of ADHF $[9,10]$ while the present study went beyond just ADHF diagnosis. Among the study subjects, dyspnea was attributed to ADHF in 43\%, COPD exacerbation in 4\%, ARDS in $7 \%$, acute pneumonia in $4 \%$, massive pleural effusion in $3 \%$, acute pulmonary embolism in $7 \%$, and AKI with volume overload and metabolic acidosis in $4 \%$. Patients having both cardiac and non-cardiac cause of dyspnea who accounted for $28 \%$ of the study population mostly had a diagnosis of ischemic cardiomyopathy (ICMP) with CKD, COPD/ILD with RV failure, and ADHF with pneumonia. In previous studies by Pirrozi et al. and Laursen et al., acute exacerbation of COPD and acute pneumonia constituted 31.3 and $30 \%$ of their 
Table 4 Characteristics of study patients according to category of diagnosis (categorical variables) ( $N=$ cardiac 43 , non-cardiac 29. Both cardiac and non-cardiac 28)

\begin{tabular}{|c|c|c|c|c|}
\hline \multirow[t]{2}{*}{ Variable } & \multicolumn{4}{|c|}{ Category of diagnosis } \\
\hline & Cardiac & Non-cardiac & Both & $P$ value \\
\hline Exertional dyspnea & 23 & 6 & 13 & .019 \\
\hline Orthopnea & 29 & 8 & 17 & .003 \\
\hline Paroxysmal nocturnal dyspnea & 9 & 2 & 3 & .204 \\
\hline Previous heart failure & 6 & 0 & 2 & .093 \\
\hline Chronic kidney disease & 0 & 0 & 4 & NA \\
\hline Chronic respiratory disease & 2 & 0 & 3 & .185 \\
\hline Coronary artery disease & 5 & 0 & 0 & .031 \\
\hline Fever and cough & 2 & 16 & 12 & .000 \\
\hline Any cardiac murmur & 1 & 0 & 1 & .616 \\
\hline Peripheral edema & 23 & 7 & 11 & .045 \\
\hline Jugular venous distension & 18 & 1 & 7 & .001 \\
\hline Displaced apex beat & 10 & 0 & 4 & .020 \\
\hline S3 & 3 & 0 & 0 & .133 \\
\hline Wheeze & 3 & 6 & 5 & .203 \\
\hline Rhonchi and rales & 0 & 2 & 3 & .110 \\
\hline Basal rales & 13 & 3 & 6 & .135 \\
\hline Rales $>1 / 3$ lung fields & 4 & 3 & 8 & .060 \\
\hline ECG abnormality & 27 & 10 & 20 & .011 \\
\hline $\begin{array}{l}\text { Lung sliding (absent } \\
\text { to decreased) }\end{array}$ & 3 & 10 & 0 & .002 \\
\hline Pleural effusion & 3 & 3 & 3 & .827 \\
\hline \multicolumn{5}{|l|}{ B profile } \\
\hline Bilateral & 31 & 12 & 20 & .07 \\
\hline Focal & 2 & 4 & 2 & \\
\hline A lines & 10 & 13 & 6 & \\
\hline \multicolumn{5}{|l|}{ EF eyeball method } \\
\hline Severe LV dysfunction & 19 & 0 & 6 & .000 \\
\hline Moderate LV dysfunction & 9 & 2 & 8 & \\
\hline Normal & 13 & 29 & 14 & \\
\hline Need for ABG analysis & 2 & 13 & 16 & .000 \\
\hline Dilated RV & 15 & 7 & 15 & .06 \\
\hline Increased kidney echotexture & 13 & 12 & 15 & .144 \\
\hline
\end{tabular}

$E C G$ electrocardiogram, $E F$ ejection fraction, $L V$ left ventricle, $A B G$ arterial blood gas, $R V$ right ventricle

study population $[11,18]$. In the PRIDE study, COPD and pneumonia constituted 25 and $10.7 \%$, respectively [23]. This difference can be attributed to the fact that most of the COPD and pneumonia patients were OP visits rather than $E D$ visits at our setting and we have not enrolled known COPD patients for whom treating physician lists no other possible diagnosis.

On logistic regression analysis of, at-admission patient characteristics, IVC diameter, EF by eyeball method, and lung sliding showed independent association between cardiac and non-cardiac diagnosis. Among the clinical variables, h/o fever and cough, and jugular venous distension showed independent association. Abnormal ECG and Boston criteria were not independently helpful to differentiate between cardiac and non-cardiac diagnosis. A study by Prosen et al. showed there is significant difference in modified Boston criteria for HF score between cardiac (mean $10.9 \pm 1.8)$ and pulmonary $(4.6 \pm 1.2)$ patients [19], while in our study, it showed little difference in scores between cardiac (mean $8.07 \pm 1.3$ ) and non-cardiac group $(6.86 \pm 1.15)$, questioning the reliability of score in differentiating HF from non-cardiac causes. This difference may be because we included patients with pulmonary, metabolic, and other systemic causes of dyspnea in our non-cardiac group in contrast to other studies which included only pulmonary as non-cardiac.

While it is well established that the presence of AIS is fairly sensitive for detecting ADHF [24], it is possible to have AIS without ADHF. B-pattern alone was not statistically significant between cardiac and non-cardiac groups as interstitial pneumonitis, pulmonary fibrosis, and ARDS will also show a similar pattern. Combined lung-cardiacIVC USG allowed us to differentiate accurately between these groups. Pleural effusion did not add to B-profile in identifying ADHF in our study as it was in LUCUS protocol study. We faced certain diagnostic challenges in differentiation between diastolic heart failure and pulmonary pathology as both can show B pattern, i.e., to distinguish between wet and dry B-lines. The entire clinical picture helped us in reaching the diagnosis such as - a hypertensive with LVH and LA enlargement with B-pattern was in favor of diastolic heart failure and normotensive with h/o chronic respiratory disease and B-pattern with dirty appearing lungs (fragmented pleural line, subpleural abnormalities) was suggestive of a pulmonary pathology.

We included renal USG in the study as the prevalence of renal failure is high in our setting. Twelve percent of our study population had kidney size of $<9 \mathrm{~cm}$. Combining renal USG to cardiopulmonary USG provided additional diagnostic data in our study population. We did not included BNP in our study as it can be elevated in the setting of CHF when an etiology other than ADHF actually accounts for the acute dyspnea and questionable economic gains and patient benefits of subjecting every patient with dyspnea to BNP assay as diagnostic uncertainty exist with mid-level BNP values $[25,26]$. One of the unique features of the present study was that previous studies have included only cardiac and pulmonary causes of dyspnea, but we have also included non-cardiopulmonary causes including renal and metabolic causes fitting more into real-world scenario. 
A significant proportion of our patients (28\%) presenting with dyspnea had both cardiac and non-cardiac cause. There is a significant increase in morbidity in these patients compared to single cause of dyspnea with increase in time to relief of dyspnea (median $36 \mathrm{~h}$ ) and hospital LOS (mean 11.5 days). So, it is important to have comprehensive search for all the major causes of dyspnea in every patient as significant number of them could be having multiple disease processes responsible for their symptoms.

Our study has limitations, the first being a small sample size. Patients were enrolled by a single physician trained in ultrasonography. All consecutive patients presenting with dyspnea to ED were not enrolled limited by the availability of enrolling physician. Because of the small sample size, some causes of dyspnea resulted in low recurrence, limiting the reproducibility of data relative to the ability of ultrasound in detecting them. We did not find pneumothorax and cardiac tamponade cases in our case series.

The ED physician sonographer could be influenced by a suggestive clinical presentation as the sonographer is not blinded to patient clinical findings. The primary endpoint was the diagnosis on the patient discharge summary. Although the analysis has been made by two independent physicians, a cardiologist and a pulmonologist, this criterion could be questionable because the final diagnosis was based on a body of evidence including ED diagnosis.

\section{Conclusions}

The present study concludes that integrating focused multiorgan USG by lung-cardiac-IVC and renal ultrasound into routine clinical evaluation for evaluating patients with dyspnea has a higher accuracy for differentiating causes of dyspnea in ED. This strategy can be adopted even in resource-limited setting with training in multiorgan USG, thereby making it widely applicable to patients presenting with dyspnea to ED. More studies are required which should include non-cardiopulmonary causes along with cardiopulmonary causes to come to reasonable decisionmaking process while evaluating dyspnea, which still remains an enigmatic symptom.

\section{Abbreviations}

ADHF: Acute decompensated heart failure; AIS: Acute interstitial syndrome; BNP: Brain natriuretic peptide; CKD: Chronic kidney disease; COPD: Chronic obstructive pulmonary disease; ED: Emergency department; EF: Ejection fraction; EPSS: E-point septal separation; ILD: Interstitial lung disease; IVC: Inferior vena cava; LV: Left ventricle; LVH: Left ventricle hypertrophy; POCUS: Point-of-care ultrasonography; RA: Right atrium; RV: Right ventricle; SD: Standard deviation; USG: Ultrasonography

\section{Acknowledgements}

The authors acknowledge Dr. Ravi Venkatachalam Chitrapu M.D., D.M, Professor of Cardiology for his suggestions and editorial assistance.

\section{Authors' contributions}

SNR-G and KV conceived the concept and design of the study, analyzed and interpreted the data, and provided the final data of the manuscript. SNR-G collected the data. All authors read and approved the final manuscript.

\section{Ethics approval and consent to participate}

This study was approved by the Rangaraya Medical College Institutional Ethics Committee, reference number IEC/RMC/2014/068, in accordance with the Declaration of Helsinki Ethical Principles for Medical Research involving human subjects. Written informed consent was obtained from all patients enrolled in the study.

\section{Consent for publication}

Not applicable

\section{Competing interests}

The authors declare that they have no competing interests.

\section{Publisher's Note}

Springer Nature remains neutral with regard to jurisdictional claims in published maps and institutional affiliations.

Received: 2 August 2017 Accepted: 23 March 2018

Published online: 04 April 2018

\section{References}

1. Logeart D, Saudubray C, Beyne P, Thabut G, Ennezat PV, Chavelas C, Zanker C, Bouvier E, Solal AC. Comparative value of Doppler echocardiography and B-type natriuretic peptide assay in the etiologic diagnosis of acute dyspnea. J Am Coll Cardiol. 2002;40:1794-800.

2. Collins S, Storrow AB, Kirk JD, Pang PS, Diercks DB, Gheorghiade M. Beyond pulmonary edema: diagnostic, risk stratification, and treatment challenges of acute heart failure management in the emergency department. Ann Emerg Med. 2008;51:45-57.

3. Liteplo AS, Marill KA, Villen T, Miller RM, Murray AF, Croft PE, Capp R, Noble $V E$. Emergency thoracic ultrasound in the differentiation of the etiology of shortness of breath (ETUDES): sonographic B-lines and N-terminal pro-braintype natriuretic peptide in diagnosing congestive heart failure. Acad Emerg Med. 2009;16:201-10.

4. Baggish AL, Lloyd-Jones DM, Blatt J, Richards AM, Lainchbury J, O'Donoghue M, Sakhuja R, Chen AA, Januzzi JL. A clinical and biochemical score for mortality prediction in patients with acute dyspnea: derivation, validation and incorporation into a bedside programme. Heart. 2008;94(8): 1032-7. https://doi.org/10.1136/ hrt.2007.128132.

5. Nazerian P, Vanni S, Volpicelli G, Gigli C, Zanobetti M, Bartolucci M, Ciavattone A, Lamorte A, Veltri A, Fabbri A, Grifoni S. Accuracy of point-ofcare multiorgan ultrasonography for the diagnosis of pulmonary embolism. Chest. 2014;145(5):950-7. https://doi.org/10.1378/ chest.13-1087.

6. Weintraub NL, Collins SP, Pang PS, Levy PD, Anderson AS, Arslanian-Engoren C, Gibler WB, McCord JK, Parshall MB, Francis GS, Gheorghiade M. Acute heart failure syndromes: emergency department presentation, treatment, and disposition: current approaches and future aims: a scientific statement from the American Heart Association. Circulation. 2010;122:1975-96.

7. Dharmarajan K, Strait KM, Lagu T, et al. Acute decompensated heart failure is routinely treated as a cardiopulmonary syndrome. PloSone. 2013;8(10):e78222.

8. Lichtenstein DA, Mezière GA. Relevance of lung ultrasound in the diagnosis of acute respiratory failure: the BLUE protocol. Chest. 2008;134(1):117-25.

9. Kajimoto K, Madeen K, Nakayama T, Tsudo H, Kuroda T, Abe T. Rapid evaluation by lung-cardiac-inferior vena cava (LCI) integrated ultrasound for differentiating heart failure from pulmonary disease as the cause of acute dyspnea in the emergency setting. Cardiovascular Ultrasound. 2012;10:49.

10. Russell FM, Ehrman RR, Cosby K, Ansari A, Tseeng S, Christain E, Bailitz J. Diagnosing acute heart failure in patients with undifferentiated dyspnea: a lung and cardiac ultrasound (LuCUS) protocol. Acad Emerg Med. 2015;22:182-91.

11. Pirozzi C, Numis FG, Pagano A, Melillo P, Copetti R, Schiraldi F. Immediate versus delayed integrated point-of-care-ultrasonography to manage acute dyspnea in the emergency department. Crit Ultrasound J. 2014;6(1):5. https://doi.org/10.1186/2036-7902-6-5.

12. Gallard E, Reddonet JP, Bourcier JE, et al. Diagnostic performance of cardiopulmonary ultrasound performed by the emergency physician in the management of acute dyspnea. Am J Emerg Med. 2015 Mar;33(3):352-8.
Funding

This is a non-funded study. 
13. Pauwels RA, Buist AS, Calverley PM, Jenkins CR, Hurd SS. GOLD scientific committee: global strategy for the diagnosis, management, and prevention of chronic obstructive pulmonary disease. NHLB/ WHO Global Initiative for Chronic Obstructive Lung Disease (GOLD) workshop summary. Am J Respir Crit Care Med. 2001;163:1256-76.

14. Remes J, Miettinen H, Reunanen A, Pyörälä K. Validity of clinical diagnosis of heart failure in primary health care. Eur Heart J. 1991;12:315-21.

15. Prosen $G$, Klemen $P$, et al. Combination of lung ultrasound (a comet-tail sign) and $\mathrm{N}$-terminal pro-brain natriuretic peptide in differentiating acute heart failure from chronic obstructive pulmonary disease and asthma as cause of acute dyspnea in prehospital emergency setting. Critic Care. 2011; 15:R114.

16. Volpicelli G, Elbarbary M, Blaivas M, Lichtenstein DA, Mathis G, Kirkpatrick AW, Melniker L, Gargani L, Noble VE, Via G, Dean A, Tsung JW, Soldati G, Copetti R, Bouhemad B, Reissig A, Agricola E, Rouby JJ, Arbelot C, Liteplo A, Sargsyan A, Silva F, Hoppmann R, Breitkreutz R, Seibel A, Neri L, Storti E, Petrovic T. International evidence-based recommendations for point-of-care lung ultrasound. Intensive Care Med. 2012;38:577-91.

17. Gargani L, Volpicelli G. How I do it: lung ultrasound. Cardiovasc Ultrasound. 2014;12:25.

18. Laursen $C B$, Sloth E, Lassen AT, et al. Point-of-care ultrasonography in patients admitted with respiratory symptoms: a single-blind, randomised controlled trial. Lancet Respir Med. 2014;2(8):638-46.

19. Beaulieu Y. Bedside echocardiography in the assessment of the critically ill. Crit Care Med. 2007;35(5):S235-49.

20. Lancellotti P, Price S, Edvardsen T, Cosyns B, Neskovic AN, Dulgheru R, Flachskampf FA, Hassager C, Pasquet A, Gargani L, Galderisi M, Cardim N, Haugaa KH, Ancion A, Zamorano JL, Donal E, Bueno H, Habib G. The use of echocardiography in acute cardiovascular care: recommendations of the European Association of Cardiovascular Imaging and the Acute Cardiovascular Care Association. Eur Heart J Cardiov Imaging. 2015;16:119-46.

21. JH MG, Cleland JG. Reliability of reporting left ventricular systolic function by echocardiography: a systematic review of 3 methods. Am Heart J. 2003; 146(Issue 3):388-97.

22. Emergency ultrasound imaging criteria compendium. Ann Emerg Med. Vo 68(Issue 1):e 11-48. https://doi.org/10.1016/j.annemergmed.2016.04.028.

23. Shafiq U. Rehman a, Abelardo Martinez-Rumayor a, Thomas Mueller b, James L. Januzzi Jr., Independent and incremental prognostic value of multimarker testing in acute dyspnea: results from the ProBNP Investigation of Dyspnea in the Emergency Department (PRIDE) study; Clin Chim Acta 392 (2008) 41-45.

24. Alan T. Chiem, MD, MPH, Connie H. Chan, MD, Douglas S. Ander, MD, Andrew N. Kobylivker, MD, and William C. Manson, MD Comparison of expert and novice sonographers' performance in focused lung ultrasonography in dyspnea (FLUID) to diagnose patients with acute heart failure syndrome. Acad Emerg Med 2015; 22: 564-573.

25. Christopher CR, Keim SM, Worster A, Rosen P; BEEM (Best Evidence in Emergency Medicine). Brain natriuretic peptide in the evaluation of emergency department dyspnea: is there a role?: J Emerg Med. 2012:42(2): 197-205. https://doi.org/10.1016/j.jemermed.2011.07.014

26. Packer M. Should B-type natriuretic peptide be measured routinely to guide the diagnosis and management of chronic heart failure? Circulation. 2003; 108:2950-3.

\section{Submit your manuscript to a SpringerOpen ${ }^{\circ}$ journal and benefit from:}

- Convenient online submission

- Rigorous peer review

- Open access: articles freely available online

- High visibility within the field

- Retaining the copyright to your article

Submit your next manuscript at $\gg$ springeropen.com 